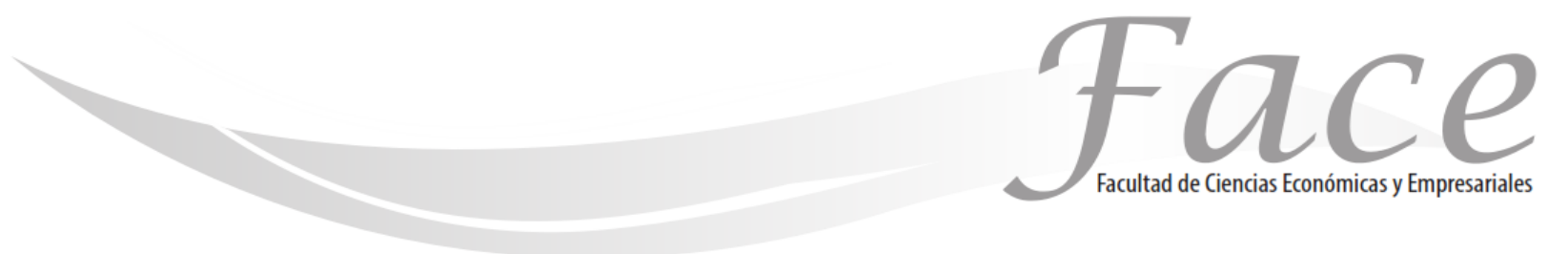

ISSN Impreso: 1794-9920 ISSN Electrónico: 2500-9338

Volumen $16-\mathrm{N}^{\circ} 1$

Año 2016

Págs. 61 - 71

\title{
LA PRÁCTICA PROFESIONAL ESCENARIO DESAFIANTE Y RESOLUTIVO
}

\author{
Clara Inés Palacios Burbano * \\ Enlace ORCID: http://orcid.org/0000-0001-8786-5127 \\ Martha Stella Bonilla Rodríguez ** \\ Enlace ORCID: http:// orcid.org/0000-0003-3821-2650
}

Fecha de Recepción: 7 de Mayo 2016

Fecha de Aprobación: 12 de Junio 2016

\begin{abstract}
Resumen:
Este artículo presenta resultados de la investigación la práctica profesional como un espacio para la duda genuina. Caracterizar las particularidades de la experiencia de práctica profesional en psicología se constituye en una tarea fundamental que posibilita reflexionar sobre la formación y los escenarios propuestos para dicho ejercicio, objetivo: Es así como el objetivo planteado en la presente investigación logra caracterizar los procesos de práctica académica de cuarenta (40) estudiantes de psicología en términos de las posibles experiencias de duda genuina que pueden surgir durante la realización de los mismos. Metodología. Se realizó un estudio de tipo no experimental y descriptivo, con diseño etnográfico, que caracteriza las experiencias de práctica que tienen los estudiantes participantes, logrando identificar las acciones dirigidas a la resolución de situaciones de tensión presentes en la práctica. Resultados: Las reflexiones que hacen los participantes giran en torno las percepciones que tienen los estudiantes en primer lugar y de manera favorable a la adecuación de su formación, concepciones acerca de la profesión elegida, sensación de preparación para enfrentarse a las problemáticas reales del mundo, las construcciones teóricas y epistemológicas creadas en el espacio académico y en segundo lugar sobre la gestión de recursos en el contexto, entendiéndolo como una estrategia resolutiva que logra construirse frente a las situaciones problema que deben enfrentar en las agencias de práctica, dónde la movilización de recursos propios permiten dar solución a la demanda del contexto.
\end{abstract}

Palabras Claves: Duda Genuina, Práctica Profesional, creencias

* Investigadora. Psicóloga Universidad Santiago de Cali. Especialista en Desarrollo Humano y Organizacional Universidad Santiago de Cali. Candidata a Magister en Psicología Social Universidad del Valle. Docente Investigadora Universidad Cooperativa de Colombia sede Cali. Contacto: clarapalacios@gmail.com.

** Psicóloga, Universidad del Valle. Maestría en Psicología Cognitiva, Investigadora Universidad del Valle Maestría en Psicología cognitiva, Docente investigadora de la universidad Cooperativa de Colombia. Contacto: marta.bonillar@ucc.edu.co 


\title{
PROFESSIONAL PRACTICE A CHALLENGING AND DECISIVE SCENARIO
}

\begin{abstract}
:
The article presents the research results of the professional practice as a space for genuine doubt. The characterization of the particularities of the professional practice experience in psychology constitutes in a fundamental task that reflects on the training and the scenarios proposed. Objective: The objective of the study was to characterize the academic practice process of forty (40) psychology students in terms of the possible experience of genuine doubt that could arise during workplace training. Methodology: A non-experimental and descriptive study, with an ethnographic design was carried out, that characterizes the students practice experience, thereby identifying their actions on how to deal with tense situations during their professional practice. Results: The reflections revolve firstly, around the perceptions that students have of their training, chosen profession, sense of preparation to confront the real problems of the world, theoretical and epistemological structures created in the academic environment and secondly, on the management of resources, understanding it as a problem-solving strategy that students face in the workplace where the mobilization of resources allows them to solve the problems that can emerge.
\end{abstract}

Keywords: Genuine Doubt, Professional Practice, Beliefs

\section{PRÁTICA PROFISSIONAL CENÁRIO DESAFIADOR E DECISIVO}

\begin{abstract}
Resumo:
Este artigo apresenta os resultados da pesquisa-prática profissional como um espaço para a dúvida genuína. A caracterização das particularidades da experiência na prática profissional em psicologia constitui-se em uma tarefa fundamental que reflete sobre a formação e os cenários propostos. Objetivo: $\mathrm{O}$ objetivo do estudo foi caracterizar o processo de prática acadêmica de quarenta (40) estudantes de psicologia em termos de experiência da dúvida genuína que possam surgir durante o treinamento no local de trabalho. Metodologia: Estudo descritivo e não experimental, com um desenho etnográfico do qual caracteriza a experiência de prática dos estudantes, identificando desse modo suas ações sobre como lidar com situações difíceis durante sua prática profissional. Resultados: As reflexões em primeiro lugar giram em torno de percepções que os alunos têm de sua formação, profissão escolhida, sentido de preparação para enfrentar os problemas reais do mundo, estruturas teóricas e epistemológicas, criadas no ambiente acadêmico e em segundo lugar, a gestão dos recursos, entendê-la como uma estratégia de resolução de problemas, que os estudantes enfrentam no local de trabalho onde a mobilização dos recursos lhes permite resolver os problemas que possam surgir.
\end{abstract}

Palavras-Chave: Duvida genuína, Prática Professional, Crença. 


\section{INTRODUCCIÓN:}

Este proyecto de investigación se planteó comprender la práctica profesional de un grupo de estudiantes de psicología, como un posible espacio para la duda genuina, la cual es entendida por Schrieber y Moss (2002) como un estado de inestabilidad, en el que las creencias que han cimentado los estudiantes con respecto a los fenómenos psicológicos se ponen en cuestionamiento frente a las experiencias y realidades inesperadas que supone la realización de una práctica académica. Teniendo en cuenta lo anterior encontramos que los estudiantes muestran diferentes estrategias para asumir las situaciones de tensión, que les demanda el escenario de práctica, pero claramente vemos que dicho escenario no se logra constituir como un espacio de duda genuina, sus relatos dan cuenta de las implicaciones que desde lo emocional, personal, académico requieren, para poder lograr dar respuesta a las demandas provenientes de las agencias de práctica, y así permitir que la intervención derive en procesos de transformación sustantivos para los pobladores y las dinámicas presentes en sus cotidianidades, pero en ningún momento se cuestiona su sistema de creencias.

El interés de realizar este proyecto de investigación tiene lugar dado que los problemas reales involucrados en la práctica académica se constituyen como fuente de formación y de articulación entre la dinámica académica y problemáticas presentes en la comunidad. La práctica profesional se constituye como un escenario de aprendizaje in situ donde el estudiante de psicología se enfrenta a realidades y problemas que le exigen tomar decisiones y realizar acciones encaminadas a abordarlos, teniendo como base una serie de conocimientos y competencias disciplinares. La práctica para Sarmiento (2009), la define como, "experiencias formativas conducente a la construcción guiada del pensamiento práctico y reflexivo" donde confluyen "tensiones y perspectivas en conflicto que inducen a la inquietud epistemológica y ontológica, en consecuencia, a una visión crítica reflexiva de su profesión"(p.21).

Desde otras áreas de formación, se puede decir que el estudio de las experiencias de práctica como estrategia formativa ha sido abordado desde diferentes miradas que confluyen en una misma idea: la posibilidad de mejorar la calidad de la experiencia formativa del estudiante y a la vez contribuir éticamente con el contexto donde desarrolla su ejercicio de formación.

\section{METODOLOGÍA:}

A continuación se presenta el aspecto metodológico de la investigación que fue de corte cualitativo, y se inscribe en el diseño narrativo, el método obedece a un estudio no experimental y descriptivo, que tiene como propósito el análisis de la experiencia de práctica profesional en psicología. El método biográfico o el análisis de relatos de vida retomo elementos de la narrativa para analizar el discurso que los participantes crearon para contar su propia historia. En este sentido, ellos se ubicaron como principales protagonista de los eventos que vivencian. De esta forma, transforma en un agente que realiza una serie de acciones que lo transforman en un ser competente, capaz de alcanzar eso que busca.

Según Cornejo (2005), en la metodología de Análisis de Relatos de Vida el sujeto se apropia de su poder de "dar forma" gracias al componente reflexivo que supone la socialización de su relato. De esta forma, el relato es narración y a la vez aprendizaje, en tanto permite al sujeto convertir su experiencia subjetiva en un objeto de conocimiento. El paso de saberes implícitos a explícitos, reconocidos y movilizables, es fundamental desde la mirada del razonamiento abductivo en tanto reconoce diferentes niveles de complejidad en las interpretaciones que se hacen de la experiencia. Lo correspondiente a participantes se logró trabajar con cuarenta (40) estudiantes de psicología, de la Universidad Cooperativa de Colombia (Cali) que se encontraban en práctica, o con su ejercicio culminado recientemente (2015), distribuidos de la siguiente forma: Diez (10) estudiantes en Práctica de Psicología Educativa, diez (10) en práctica de Psicología Clínica, Diez (10) estudiantes en Práctica de Psicología Organizacional y diez (10), con su ciclo de formación recientemente concluido. La selección de los participantes responde a la condición de matrícula activa en las prácticas y a la disposición y compromiso de los estudiantes con las actividades involucradas en la investigación. Los instrumentos utilizados para recopilar la información fueron a través de grupos focales - método biográfico (análisis de relatos de vida), en primer lugar el grupo focal se propuso analizar los relatos contados por los estudiantes durante los grupos focales, (todos los encuentros fueron grabados en audio y luego transcritos), referentes a las experiencias y anécdotas que enmarcaron la experiencia de práctica.

El contenido de las narraciones se logró analizar por medio del programa ATLAS-TI 7, versión libre, posibilitando la identificación cinco categorías emergentes, (se presentan en orden descendente): Limitado acompañamiento del asesor institucional, Tiempo limitado para la intervención, Dificultad en el apoyo psicológico, Dificultad en la articulación de los tiempos de la Agencia y la Universidad, Rol indefinido del psicólogo practicante. 
Por último se realiza la interpretación de la información espacio en el que logran construir las descripciones explicativas gracias la relación entre las categorías iniciales, y las emergentes, dando como resultado la aproximación nuevos discernimientos, en torno al escenario de práctica, para ello fue indispensable articular los relatos de los participantes y los autores.

\section{RESULTADOS}

Los hallazgos realizados durante este ejercicio, permitieron registrar lo que sobreviene con las creencias de los practicantes, la forma como construyen soluciones posibles en el proceso de la práctica profesional, y la relación que se establece con su lugar de práctica (agencia), la población, asesor/a institucional, Asesor/a Académico/a, compañeros/as de práctica y Universidad en la cual circunscribe.

El análisis que se realizó de la práctica a partir de la aplicación de los instrumentos empleados por la investigación permitió la reflexión en torno a las categorías iniciales (Pertinencias de los conocimientos teóricos, Evaluación herramientas metodológicas (intervención), Evaluación del proceso de seguimiento y monitoreo de la intervención, Acompañamiento asesores, Relación Universidad- Agencia-Practicante, Problemáticas recurrentes y Estrategias resolutivas de los practicantes y emergentes, (Limitado acompañamiento del asesor institucional, Tiempo limitado para la intervención, Dificultad en el apoyo psicológico, Dificultad en la articulación de los tiempos de la Agencia y la Universidad, Rol indefinido del psicólogo practicante).

Con relación a la pertinencia de los elementos teóricos para enfrentar la práctica, el total de los participantes perciben que saben cómo emplear los conocimientos y las herramientas disciplinares que conocen para el diagnóstico, planeación y ejecución de intervenciones centradas en necesidades psicológicas detectadas, esta certeza se la atribuyen principalmente a la fundamentación teórica y práctica que han recibido a lo largo de la carrera y a la preparación ofrecida por los asesores académicos. En los casos de los estudiantes con más de un nivel de práctica, una tercera atribución corresponde a la experiencia adquirida con los beneficiarios en semestres anteriores.

Frente a monitoreo y seguimiento en procesos de intervención, los participantes manifestaron dudar parcialmente de sus conocimientos para monitorear 0 hacer un seguimiento detallado a los avances o retrocesos de los beneficiaros al interior de sus proyectos de intervención. Esta situación resultó especialmente particular para los estudiantes de último año de psicología, los niveles de práctica ocasionaron cambios de población y agencia, y frente a ello aducen que en cuatro meses no se alcanzan a evidenciar resultados, esto dificulta el seguimiento a los procesos, (36 practicantes). Frente a ello los estudiantes que permanecen en una misma agencia, (4 practicantes), explicitan haber desarrollado estrategias para hacer este seguimiento y para registrar evidencias de avances.

Por otro lado, el grupo focal desarrollado con los practicantes de segundo ciclo de práctica 10 participantes (Educativa), manifestaron no sentirse seguros para realizar una evaluación de su propio proceso, algunas situaciones que hacen a los estudiantes dudar de esos conocimientos fueron atribuidas a la insuficiente preparación 0 acompañamiento ofrecido por los asesores institucionales. (Véase figura 1), las personas encargadas del acompañamiento en campo resultaban poco disponibles 0 poco preparadas para dicha tarea. El fallo en el asesor institucional se adjudicó a una falta de comunicación entre las agencias de práctica y la Universidad. A esta última no se le reportan los cambios institucionales al interior de la agencia, por ello enlistan la necesidad de monitoreo continuo y exigencia en el perfil de los asesores institucionales.

Los estudiantes también mencionan que los pocos espacios de discusión con compañeros del mismo ciclo o de ciclos superiores, puede afectar su percepción de seguridad sobre conocimientos teóricos y prácticos para el diseño, ejecución, seguimiento y evaluación de los proyectos de intervención

El $78 \%$ de los estudiantes manifestaron haber experimentado altos niveles de angustia y $22 \%$ de ellos afirmaron haberse sentido alguna vez desbordados por las demandas académicas mezcladas con las situaciones vividas durante la práctica. Sin embargo, $98 \%$ de ellos manifestaron haber podido regular sus emociones.

La angustia fue atribuida principalmente a la recepción negativa 0 indiferente de las actividades por parte de los beneficiarios y a la acumulación de carga académica asignada por los profesores del semestre. La segunda atribución para ambas emociones se relacionó con la falta de apoyo o el incumplimiento de los compañeros de trabajo con los compromisos de práctica. Una tercera razón fue la retroalimentación desfavorable o inexistente, brindada por los asesores institucionales y los trabajos inesperados asignados por éstos y asesores académicos, (Insuficiente planeación, comunicación deficiente Universidad -Agencia en cuanto a cronogramas de trabajo). 
La posibilidad de regular las emociones se atribuyó a fortalezas personales, una dinámica de trabajo positiva entre compañeros y el apoyo de asesores académicos.

El $67 \%$ de los participantes mencionó que en algún momento de la práctica tuvo la sensación de no saber qué hacer, en su proceso de intervención, las situaciones con las que esto se asoció fueron, principalmente, la falta de experiencia y herramientas para resolver situaciones presentadas en la práctica, la dificultad para conectarse con la población.

La falta de apoyo del asesor institucional en términos de rutas puntuales de acción, como lo menciona uno de los participantes:

"allá carecimos de un asesor institucional, dese el primer día que llegamos allá éramos como perdidos no sabíamos a quién dirigirnos, comenzamos a preguntar a otra estudiante practicante que estaba haciendo su práctica clínica que ya conocía los lideres allá, ella fue la que nos ayudad a contactar con los líderes comunitarios nosotros en un momentos nos sentimos como cuestionados y sentimos un poco de molestia hacia la universidad, porque nos sentimos abandonados" (Grupo focal 10 de mayo de 2015).

A pesar de lo anterior, el 75\% afirmó reconocer el valor de lo construido durante la carrera en términos de su pertinencia y utilidad, es decir que no dudaron de que contaban con esos conocimientos. La dificultad que se presenta es procedimental, no se duda del valor de los conocimientos construidos ni transmitidos, sino de cómo y cuándo usarlos.

El $95.4 \%$ de los estudiantes piensa que la práctica lo ha motivado más por la carrera de psicología, especialmente porque logran identificar cambios significativos en la población y porque han logrado identificar experiencias importantes que los han ayudado a desarrollar herramientas para el trabajo de intervención (aunque no los puedan medir, ya que no saben cómo articularlo al conocimiento disciplinar) Los estudiante además reconocen cambios en sí mismos movilizados por la interacción con la población. Empiezan a otorgar un valor diferente y de importancia a la lógica y el saber de la población, al contexto y a las dinámicas que en este se suscitan. Manifiestan además que los cambios en sí mismos se articulan con resultados positivos en la población.

El $75,4 \%$ afirma que la experiencia de práctica ha reafirmado su elección de carrera. Los estudiantes que han dudado de su elección lo han hecho debido a dinámicas de trabajo entre pares percibidas como negativas.

La mayoría de los estudiantes mencionan ser capaces de promover la participación activa y la cooperación en otros. Sin embargo, indicaron estar parcialmente de acuerdo con su capacidad para intervenir en crisis. El desarrollo de las capacidades mencionadas es atribuido a la fundamentación teórica y práctica que han recibido durante la carrera y a la experiencia adquirida a partir del contacto con la población en prácticas anteriores. Por el contrario, los que dudan de estas capacidades, asocian sus dificultades con pocas experiencias de vida que los prepare para asumir el trabajo con personas con características y necesidades diferentes. Además, el poco apoyo del asesor institucional también repercute en la evaluación que el estudiante hace acerca de sus propias habilidades interpersonales.

En contraposición a la dificultad identificada por asesores académicos y profesores, pocos estudiantes dudan de sus competencias de escritura y lectura. Centran el dominio de estas habilidades, especialmente las orales, en características de su personalidad como extroversión, cordialidad o carisma. Aquellos que dudan también atribuyen a la personalidad sus dificultades, además de la preparación insuficiente en el colegio o pocas actividades universitarias que las promuevan.

\section{DISCUSIÓN:}

Este apartado lo dedicaremos para reflexionar acerca de los relatos de los estudiantes, las cuales giraron fundamentalmente en torno a tres aspectos el primero, hace referencia al lugar de los estudiantes en el escenario de práctica, el segundo a las corresponsabilidades en la formación profesional y el tercero la gestión de los recursos en el contexto, los cuales presentamos a continuación:

1. El lugar de los estudiantes en el escenario de práctica profesional La práctica como lugar de aprendizaje puede entenderse desde la cognición situada y el aprendizaje significativo, así desde esta perspectiva es preciso hablar de un escenario con realidades y desafíos permanentes para los practicantes. Según Wenger (1998), los escenarios de práctica son sistemas sociales donde podemos aprender, los cuales están conformados por personas que tienen un vínculo entre sí gracias a tareas, saberes, bienes que comparten, escenarios variados que son negociados por la comunidad a través del tiempo. Según Orozco, J. \& Bustamante, A. R. (2014) son espacios en los cuales se participa de manera diversa y 
donde además se adquieren conocimientos múltiples en niveles diferentes.

Al interior de los escenarios de práctica los roles que se presentan entre los diferentes miembros, son múltiples, claro ejemplo de ello lo podemos identificar en el lugar asignado y asumido de los practicantes, quienes se auto-clasifican en novatos y expertos, (denominando así a quienes inician su experiencia y quienes llevan más de un periodo semestral en la agencia de práctica), para el nivel de novatos, uno de los participantes menciona de su experiencia en la práctica social,

"...entonces toco en un principio dejar las formalidades a un lado las actividades académicas y buscar técnicas para poder entender cuáles eran las concepciones que ellos tenían y ajustarlas también a nuestro proyecto, nos tocó, en el principio yo diría que bastante duro, porque de hecho nos generó mucha frustración que en varias sesiones nosotras preparábamos los mega talleres y hacíamos cosas que para nosotros eran súper espectaculares y la profesora nos decía si muy bien, pero cuando llegábamos allá no había con quien aplicarlos, entonces fue frustrante entendimos que no eran nuestras concepciones que tenían que primar, sino las de ellos y era nuestro proyecto el que debía ajustarse a lo que ellos nos estaban ofreciendo". (Grupo focal 20 de mayo de 2015).

De acuerdo a lo mencionado por Gallo (2014) este grado de experticia determina la interacción con los demás y como se asume dentro de esa dinámica, se establecen aprendizajes con relación, a sí mismos, a la agencia, a los asesores, a las problemáticas que tienen lugar en el contexto de práctica, a las acciones resolutivas que asumen frente a los desafíos que la misma les presentan.

Segùn Ahmad Jamil, N., Shariff, S. \& Abu, Z. (2013), el novato escala un nuevo lugar resultando con ello que la relación estudiante-asesor, se transforma, pudiendo llegar a tomar el lugar de relación entre pares, resultado de dicha transformación, la identidad se transforma y produce que el practicante configuren sus acciones de diferente manera y puedan descifrarse a sí mismo desde un nuevo lugar, pero a su vez en la comunidad también se dan cambios, es un sistema y termina siendo afectado en su totalidad.

Se debe propender porque la experiencia del practicante sea retadora y le permita avanzar, de manera que puede ubicarse en los diferentes escenarios que se requieren para perfeccionar su nivel de experticia, entendiéndose como un aprendiz que requiere de tiempo y una suficiente claridad epistemológica, teórica y práctica, dicho ejercicio.

Concluyendo este primer aspecto se logra comprender que el nivel de novato o experto, está dado en la medida que el practicante tiene o no claridad y dominio sobre el proceso de intervención. La relación entre el modelo pedagógico donde se contiene la práctica y la sensación de preparación frente a los problemas del mundo real (Bueno Millan, Semer, Da Silva Rodríguez, y Gianinni, 2012), deben ser directamente proporcionales, para que el estudiante logre contar con algunas certezas que requiere al enfrentarse al nuevo escenario y al mundo laboral formal. Si bien el grupo de participantes reconoce algunas limitaciones mínimas en términos de su proceso formativo, logran identificar como una necesidad urgente, de acuerdo a Benatuil, D., \& Laurito, J. (2015) la cual emerge como observación realizada por los participantes, lo concerniente a la intervención, dado que actualmente la intervención se aborda en séptimo semestre, paralelamente con el inicio de ciclo de práctica social comunitaria ${ }^{1}$, por lo cual reflexionan en torno a la pertinencia que este momento se genere previo a su llegada a la práctica, como una forma de prepararlos para el momento de inicio del ciclo.

\section{Corresponsabilidades en la formación} profesional Según Alcantar y Arcos (2003) indican que la práctica profesional es fundamental por el vínculo diádico de escenarios donde el estudiante puede articular teoría y práctica, dichos escenarios se favorecen de manera bilateral (Laboral y Académico), en primer lugar, por la posibilidad que las agencias se actualicen en lo correspondiente a la intervención y el trabajo articulado con una institución de formación superior que visibiliza aún más su labor e imagen, y como valor agregado el poder contar con un equipo de trabajo constituido por practicantes y profesionales que acompaña el proceso y que beneficia la consecución de sus metas.

Desde la Universidad, ya que le permite dar cumplimiento a su objetivo de formar profesionales calificados que posteriormente logren hacer inmersión en el mundo laboral, logrando así concluir su ciclo de formación, perfeccionando

$1 \quad$ En la entidad de educación superior, (Universidad Cooperativa de Colombia, sede Cali), donde se llevó a cabo la presente investigación, el modelo de práctica se desarrolla en cuatro niveles, comprendidos de la siguiente manera: VII semestre pre-practica comunitaria, VIII práctica Educativa, IX práctica Clínica y X práctica organizacional. 
los aspecto teóricos abordados durante los semestres de formación teórica, sumado a ello se puede mencionar también el cumplimiento a los requerimientos del orden nacional del ministerio de educación (MEN), los cuales indican que la entidad está en obligación de brindar espacios de formación propicios para el futuro profesional.

Los participantes en la presente investigación, reconocen que los escenarios en los cuales desarrollan su práctica deben contar con unas características mínimas que posibiliten el aprendizaje necesario para cada ciclo, como lo menciona un practicante, que concluyó su ciclo de formación, "Hay agencias de agencias, yo concluí todo el proceso y puedo afirmarlo, en mi recorrido me encontré con algunos lugares, que no forman sino que deforman, y otros en los cuales, uno puede decir que aprendió" (Grupo focal, 19 de mayo del 2015).

Resulta imprescindible realizar una lectura crítica de las agencias, evaluando los requerimientos y el nivel de cumplimiento que se le da a los mismos, dentro del contacto inicial y los compromisos consignados entre agencia y universidad se cumple con lo solicitado, pero la realidad que experimentan algunos de los practicantes es otra, según Alpert, F., Heaney, JG.,\& Khun, KA (2009), concluyen que se debe hacer una revisión a los programas de práctica teniendo en cuenta las relaciones tejidas entre las tres instancias involucradas en ellos (la universidad, la agencia de práctica y los practicantes). Una adecuada revisión y análisis permitiría construir una base común para el beneficio mutuo de las tres instancias.

El acercarse a la agencia de práctica desde una mirada formativa implica que se verifique si dicho escenario cumple con una los requisitos que la constituyen como una entidad formadora propicia para los futuros profesionales. Arias (2003) el vínculo que se establece debe ser claro y brindar las posibilidades de formación contempladas desde el plan curricular, evitando situaciones que van en detrimento del proceso., según lo menciona un practicante de clínica, frente a su inicio con la práctica comunitaria, "nosotros fuimos pioneros en práctica social allí, en cierta medida eso fue bueno, pero a la vez tiene sus proceso en contra, entonces una situación compleja que note en la práctica social fue que entre la universidad y la agencia no hubo una comunicación asertiva, ósea nunca se comunicaron en realidad". (Grupo focal 20 de mayo del 2015), otra de las situaciones presentes identificadas, en dicha relación según un aprendiz, "las dificultades que siento que fueron como muy marcadas inicialmente es que no hay una comunicación entre el asesor académico y el institucional" (Grupo focal 19 de mayo del 2015)

Assante, Huffman y Harp, (2010; citados por Kim y Park, 2013) afirman que una guía clave entorno a la alta calidad de un programa académico es la presencia de un lugar de práctica formativa adecuado. Esto obedece a que el estudiante puede articular sus conocimientos con una experiencia real, dónde logra transformar y ser transformado.

El estudio de las experiencias de práctica como estrategia formativa ha sido abordado desde diferentes miradas que confluyen en una misma idea: la posibilidad de mejorar la calidad de la experiencia formativa del estudiante (Kim y Park, 2010; Bonilla, 2009; Peirce, 1903). Como un objetivo fundamental que debe plantearse la academia como entidad responsable de la formación de profesionales formados de manera ética.

\section{Gestión de recursos en el contexto}

Durante el desarrollo de la práctica cada aprendiz se ve enfrentado a diversas problemáticas, las cuales se logran identificar y clasificar gracias a las narraciones de los participantes estableciendo una escala ascendente de mayor frecuencia menor frecuencia, a continuación se presentan de manera detallada cada uno de los cinco puntos identificados por los participantes:

1. Limitado acompañamiento del asesor institucional. Los estudiantes en práctica hacen énfasis al aspecto relacionado con el acompañamiento, y la comunicación con sus asesores institucionales, reconociéndolo como una de las dificultades más frecuentes a la que deben hacer frente en la práctica, le atribuyen esta dificultad predominantemente a la carga laboral del asesor en su lugar de trabajo, en menor nivel de frecuencia a la rotación laboral del cargo, incapacidades médicas 0 campo disciplinar diferente.

2. Tiempo limitado para la intervención. Está ligada a los tiempos estipulados para desarrollar la práctica, (ejercicio de impresión diagnóstica, elaboración de la propuesta, presentación de proyecto, desarrollo de la intervención y retroalimentación de los informes, informe final).

3. Dificultad en el apoyo psicológico. A pesar de que algunos estudiantes de práctica asistieron a un número reducido de sesiones de psicoterapia que les permitió asumir la práctica desde otra perspectiva, todos los participantes manifestaron la necesidad del apoyo del psicólogo continuo durante el proceso, como un ejercicio fundamental para garantizar el bienestar de sí mismo y de la población intervenida.

4.

5. Dificultad en la articulación de los tiempos de la Agencia y la Universidad. Está relacionado con la concordancia en los procesos tanto en las agencias, como en la Universidad, ya que cada 
una maneja ciclos de tiempo diferentes, anual y semestralizado, igualmente está ligado a la variable del tiempo y de los canales de comunicación dispuestos entre ellos, sobre los cuales mencionan deben ser mejorados.

6. Rol indefinido del psicólogo practicante. Se identifica como dificultad, toda vez que se le asignan funciones que no competen a su lugar como practicante, 0 de manera más puntual, desconociendo las competencias que debe desarrollar en el ciclo que le corresponde, (comunitario, educativo, clínico, organizacional), por lo que en determinados casos, las expectativas de la agencia están en relación a una práctica diferente a la que se encuentra el estudiante.

Frente a esta serie de dificultades los estudiantes identifican la gestión de recursos en el contexto, como la estrategia empleada por todos los participantes. Es en este apartado que se aborda de manera directa el interés inicial de la investigación en torno al posible surgimiento de la duda genuina en la práctica profesional en psicología, posteriormente se exploran los recursos que el practicante auto gestiona en el contexto de práctica como una respuesta a las dificultades identificadas.

Al respecto de la duda genuina, se retoma a Schrieber y Moss (2002) quienes realizaron un estudio donde analizan las diferentes estrategias que emplearon un grupo de docentes, a la luz de lo que ellos denominaron duda genuina. Entendida como lo que debía asumir el docente frente a una situación problema que no tenía precedentes, lo cual implicaba que sus conocimientos y creencias eran inadecuados e insuficientes. Gracias a esa inesperada situación se formaba un movimiento investigativo y reflexivo, que conducía a la revisión y replanteamiento de saberes y creencias, de igual forma a la búsqueda de información y a dialogar con otros docentes, además a plantearse relaciones nunca antes consideradas.

Al hacer el análisis respectivo de las narrativas de la población nos encontramos con las acciones que realizan los practicantes, no corresponden a los requerimientos mencionados por los autores, ya que no se cuestiona su sistema de saberes, sino que dan cuenta de un ejercicio de autogestión emprendido por cada aprendiz, según Sarmiento (2009), para lograr asumir los desafíos que le presenta la práctica, dichos movimientos de autogestión se presentan en seis pasos progresivos, comprendidos como acciones resolutivas, (Apoyo en compañeros, Apoyo del asesor académico o institucional, Cursos y seminarios solicitud de bibliografía, Empatía , Apoyo en los docentes de asignaturas relacionadas con la práctica) ${ }^{2}$, que les permite construir salidas posibles frente a la situación problema del escenario de práctica, donde la solidaridad ${ }^{3} y$ la autogestión son elementos constituyentes, a pesar de ser numerosos pasos, no alcanzan a tener el carácter de la duda genuina, dado que su sistema de creencias no es cuestionado. Gómez (2009), Solo desarrollan habilidades que les permite avanzar en el ejercicio de práctica de manera segura, mencionan un estado de tensión, propio del inicio de la experiencia, pero ninguna de las narrativas da cuenta de un cuestionamiento de sus creencias, dentro de los pasos mencionados por los practicantes, encontramos:

1. Apoyo de compañeros: Consultar con compañeros de la agencia sobre la situación frente a la que se tiene duda, o se requiere intervenir, (en la mayor parte de los casos, se obtiene respuesta colaborativa frente a lo planteado).

2. Apoyo del asesor académico Consultar a su asesor/a académico y si la respuesta no posibilita la solución de manera eficaz, se acude a un profesor que esté a cargo de alguna asignatura del área de práctica.

3. Bibliografía Realizar una revisión bibliográfica, en su totalidad de preferencia consulta virtual.

4. Empatía Quienes cuentan con experiencia laboral previa, la emplean como recurso para dar solución a la situación pendiente, articulando experiencia laboral con la situación problema de práctica.

5. Asesor Institucional Como último recurso se acude al asesor/a institucional, dentro de lo que aducen para enlistarlo como último recurso, encontramos, la falta de tiempo del mismo, una relación distante, el conocimiento limitado que se evidencian en la cotidianidad del asesor, y en algunos casos incluye faltas a la ética profesional.

Los participantes no hacen este recorrido de búsqueda, por una crisis en su sistema de creencias, sino como una forma de asumir una nueva tarea. Como lo afirma uno de los participantes, "uno busca la forma de resolver las

\footnotetext{
2 Autogestión resolutiva en el contexto de práctica

3 "acto genuino que convoca a pensar en los otros, en lo que le acontece a la gente y en lo que sucede en el territorio que habitan, no esperando retribuciones de tipo monetario o social, proponiendo no sólo las ideas y soluciones a una circunstancia específica sino gestionando los recursos para resolverla". Echeverry, L y Palacios, C (2014) sin publicar.
} 
dudas, comienza preguntando a los más cercanos y así sucesivamente, también consulta en internet, sobre experiencias similares que le permitan intervenir y solucionar lo que uno necesita en el momento", (grupo focal 19 de mayo del 2015).

Para finalizar retomamos la definición de duda genuina según Schierber y Moss (2002), es "un estado en donde los conocimientos y las herramientas construidas en la academia se reconocen como insuficientes frente a un escenario novedoso que las pone a prueba" (pág.23 ); de acuerdo a lo antes mencionado, uno de los participantes afirma:"recurrir a saberes adquiridos durante el proceso de formación...pero durante el proceso de nuestra carrera hemos visto ciertas asignaturas que nos han servido 0 dado algún aporte para tenerlo en cuenta en el proceso de las prácticas" (grupo focal 18 de mayo del 2015); es decir que en los practicantes no hubo un estado de inestabilidad en donde los conocimientos que tienen fueran faltos para las realidades inesperadas 0 situaciones que se les presentaron.

Es decir, que las experiencias y situaciones que tuvieron en el trascurso de la práctica académica no ponen en duda el conocimiento que ellos ya han construido, sino que la práctica les proporciona enfrentarse a fenómenos de la vida real, aplicar como transferir significativamente el conocimiento que han adquirido, manejar situaciones sociales, contribuir a su comunidad y desarrollar en ellos competencias profesionales; de acuerdo a lo mencionado, una participante afirma que la práctica fue para ella como un proceso terapéutico "Bueno fue más un proceso como terapéutico en la práctica, darme cuenta de muchas cosas de uno mismo" (Grupo focal 19 de mayo de 2015).

Por consiguiente, lo que se halla en los relatos de los participantes, es que la práctica académica les genera sentimientos de angustia, miedo, temor, ansiedad, preocupación, entre otras emociones frente a todos aquellos episodios que viven realizando su práctica, de esta manera uno de los participantes menciona: "Bueno, o sea, eran muchos miedos lo que uno tenía. Entonces esa fue una de las principales dificultades, la angustia que a uno le genera eso" (grupo focal 18 de mayo del 2015), en otras palabras los estudiantes llevan consigo muchas inquietudes, las cuales aunque no son reflejadas, ni expresadas no les afecta directamente en la ejecución de sus prácticas.

Es decir, que los estudiantes cuando se acercan a realizar su práctica académica, tienen muchas expectativas, preguntas y preocupaciones referentes a cómo van hacer y qué cosas van a vivenciar en ellas, de acuerdo a lo anterior, en otras palabras se muestran temores que puede experimentar cualquier estudiante próximo a enfrentarse a experiencias de practica dentro de su formación académica.
A manera de cierre se retoma a Echeverri (2014) quien plantea que: "Las preocupaciones en los estudiantes próximos a comenzar su trayectoria práctica hacen alusión a todo aquello que les genera inquietud, intranquilidad 0 temor frente a su futuro rol como practicantes" (pág.2). Es decir que todos esos sentimientos se desencadenan debido a que los practicantes piensan si van a responder de manera eficiente frente las demandas que conlleva la práctica académica 0 si se les va a dificultar llevar este proceso, a los practicantes no solo les preocupa su eficacia en la realización de la práctica, sino también, como la comunidad en donde están interviniendo los va a recibir y va a responder.

\section{CONCLUSIONES:}

Dentro de lo encontrado podemos afirmar entonces que la experiencia de práctica, contrario a la hipótesis inicial y propósito fundamental de la presente investigación, surgimiento de la duda genuina, no se presenta, dado que finalmente los estudiantes construyen rutas de búsqueda alternativas para dar solución a lo que emerge en la práctica, es decir a problemáticas reales de la comunidad en la cual intervienen, quedando frenado la posibilidad de que la duda genuina aparezca, su sistema de creencias en primer lugar no entra en crisis, solo en estado de tensión, puesto que afirman que confían en el proceso formativo inicial brindado por la Universidad en la cual están circunscritos y en segundo lugar, logran dar solución a situaciones problema con sus propios recursos, lo que les permite autogestionar y solucionar de manera eficaz.

Por otro lado uno de los aportes sustanciales que realiza la presente investigación, para la formación de los practicantes de la Universidad Cooperativa de Colombia, se relaciona con las técnicas de intervención, ya que se sienten poco preparados en este aspecto, y ello genera un alto nivel de tensión al momento de iniciar la práctica (prepráctica social), por ello la población sugiere una revisión en el pensum que posiblite incluirlo en semestres de formación inferiores a la práctica 4

Otra de las conclusiones importantes que se logra presentar aplicable para las instituciones formadoras, hace referencia a la importancia del seguimiento que se debe realizar a los escenarios de práctica, donde los estudiantes circunscriben su ciclo formativo, dicho seguimiento debe propender por el cumplimiento de los compromisos adquiridos, como un factor fundamental en la formación de 
ISSN: 1794-9920 - Enero - Julio

los practicantes, pero además por el compromiso ético con la población donde se desarrolla la intervención.

\section{REFERENCIAS:}

Ahmad Jamil, N., Shariff, S. \& Abu, Z. (2013) Students' Practicum Performance of Industrial Internship Program. Procedia. Social and Behavioral Sciences, 90, 513-521.

Alpert, F., Heaney, JG.,\& Khun, KA (2009). Internships in marketing: Goals, structures and assessment - Student, company and academic perspectives. Australasian Marketing Journal (17) 3645

Andreozzi, M. (2011). Las prácticas profesionales de formación como experiencias de pasaje y tránsito identitario. Archivos de Ciencias de la Educación, 5(5). Disponible en: http://www.archivosdeciencias.fahce.unl p.edu.ar/article/view/ARCHv05n05a09

Alpert, Heaney y Khun (2009) A comparative analysis of pre-and in-service teacher beliefs about readiness and selfcompetency: Revisiting teacher education for ELLs. System, 38, 228244

Alcantar Enríquez, V. \& Arcos Vega, J. (2004) La vinculación como instrumento de imagen y posicionamiento de las instituciones de educación superior. Revista Electrónica de Investigación Educativa 6 (1). Recuperado el 2 de abril de 2013 en http://redie.uabc.mx/vol6no1/contenidoenriquez.html

Assante, Huffman y Harp, D.J. (1996). Modeling the six modes of Peircean abduction for educational purposes. Documento presentado en la Annual Midwest Reunion of $\mathrm{Al}$ and Cognitive Science Conference, Bloomington, IN. Recuperado el 25 de Mayo de 2009. Disponible

en http://www.cs.indiana.edu/event/maics9 6/Proceedings/shank.html
Arias, J. (2003). ¿Por qué los alumnos no construyen un conocimiento psicológico académico y profesional integrado?: reflexiones para una investigación necesaria. Papeles del psicólogo, (86), 5. disponible en:http://dialnet.unirioja.es/servlet/articul o? codigo $=870664$

enatuil, D., \& Laurito, J. (2015). El rol de las prácticas profesionales supervisadas en una muestra de estudiantes de psicología de una universidad de gestión privada argentina. PSIENCIA: Revista Latinoamericana de Ciencia Psicológica, 7(2), 6.

Bonilla Rodríguez, M.S. (2009) Razonamiento abductivo de niños de 10 años al interpretar un texto narrativo. Tesis de Maestría. Universidad del Valle

Bueno Millan, L.; Semer, B., da Silva Rodriguez, J.M \& Gianini, R.J (2012) Traditional learning and problem-based learning: self-perception of preparedness for internship. Revista Associacion Medica du Brassil; 58(5) 594-599

Cornejo (2006) El Enfoque Biográfico: Trayectorias, Desarrollos Teóricos y Perspectivas. Psykhe, 15 (1) 95-106

Echeverri (2014): (1996) Peirce y Ortega. Traducción de Eduardo Lostao. Universidad dePollat Navarra. Recuperado el 13 de Febrero de 2012 en:

http://dspace.unav.es/dspace/bitstream/ 10171/534/5/6.\%20PEIRCE\%20Y\%200 Peirce, C.S (1903). On Three Types of Reasoning (CP 5. 151-179). En: J. Vericat (tr., intr. y notas) (1988). Charles S. Peirce. El hombre, un signo (El pragmatismo de Peirce.) Barcelona: Crítica. Recuperado el 3 de diciembre de 2012. Disponible en http://www.unav.es/gep/OnThreeTypes Reasoning.html

Erausquin, C., Basualdo, M. E., Btesh, E., Lerman, G., Bollasina, V., \& García Coni, A. (2005). Categorías de análisis de los" modelos mentales" que construyen los psicólogos en formación en" comunidades de aprendizaje y 
Clara Inés Palacios Burbano - Martha Stella Bonilla Rodríguez

práctica. Disponible en $<$ http://www.scielo.org.ar/scielo.php?scr ipt=sci_arttext\&pid=S1851$16862005000100005 \&$ Ing=es\&nrm=iso >. ISSN 1851-1686

Erausquin, C., Basualdo, M. (2006). Heterogeneidad de giros y tensiones en los modelos mentales de" psicólogos en formación" sobre la intervención profesional en problemas situados: Hacia una dialéctica de la diversidad. Anuario de investigaciones, 13, 105123. disponible en: http://www.scielo.org.ar/scielo.php?pid= S1851-

16862006000100011\&script=sci_arttext

Gallo, C. E. (2014). La práctica profesional y sus preocupaciones. Poiésis, @ (28). Disponible en: http://www.funlam.edu.co/revistas/index .php/poiesis/article/view/1400

Gómez, C. M.(2009) Un re-pensamiento de la noción de práctica profesional. Disponible en: http://148.206.107.15/biblioteca_digital/ articulos/12-399-5934vib.pdf

Niño, D. (2009). Algunas reflexiones sobre la duda y la creencia. Cuadernos de Sistemática Peirceana, 1, 159-180. disponible en: http://www.researchgate.net/profile/Dou glas_Nino/publication/232607878_Algu nas_reflexiones_sobre_la_duda_y_la_c reencia/links/Ofcfd508708089b1900000 00.pdf

Orozco, J. \& Bustamante, A. R. (2014). La práctica profesional en el Programa de Psicología de la Fundación Universitaria Luis Amigó: Entre el ser y el hacer. Poiésis, @(28). Disponible en: http://www.funlam.edu.co/revistas/index .php/poiesis/article/view/1383

Sarmiento, L. (2009) La práctica profesional como espacio de investigación con base en las narrativas. Acción pedagógica,18, 20-29
Schreiber, J.B \& Moss, C (2002) A Peircean View of Teacher Beliefs and Genuine Doubt. Teaching \& Learning, 17 (1) 25-426 